\title{
Análisis de la perspectiva de género en normativas educativas. Comparativa de estudio de casos escolares de infantil y primaria.
}

\author{
Rodríguez-Casado, María del Rocío \\ Universidad Pablo de Olavide, Sevilla, España \\ mrrodcas@upo.es
}

\section{Resumen}

La perspectiva de género suscita un gran interés en y para la sociedad, especialmente cuando se enmarca en el ámbito educativo. No cabe duda que dicha temática sigue presentada hoy en día como un reto social, cultural, político... pero, fundamentalmente, educativo. Pues, tenemos presente que, cada vez más, nos encontramos con alumnado de diferentes realidades, experiencias y situaciones con los que debemos trabajar de manera inclusiva, integradora, igualitaria y equitativa. En este sentido, trabajar desde la perspectiva de género en las escuelas hace que nos cuestionemos los distintos estereotipos de roles culturales con los que hemos crecido y hemos sido educados/as, así como nos abre la posibilidad de elaborar nuevos o mejorar los existentes contenidos de socialización y de relación entre la personas en pro de la igualdad. Por ello, nuestra investigación gira en torno al análisis de la perspectiva de género reflejada en los proyectos educativos de cuatro centros escolares públicos, desde una mirada comparativa, con la finalidad de identificar qué modelo de atención a la perspectiva de género está presente en sus normativas educativas, a través de un análisis documental de contenido como metodología cualitativa de investigación. En definitiva, este estudio nos permite acercarnos a realidades determinadas para mejorar la calidad de la práctica docente, avanzar hacia los fines de una educación intercultural con perspectiva de género y transformar el rol que ocupa la escuela en nuestra sociedad plural.

\section{Abstract}

The gender perspective arouses great interest in and for society, especially when it is framed in the educational field. There is no doubt that this theme is still presented today as a social, cultural, political... but fundamentally educational challenge. Well, we keep in mind that, increasingly, we find students from different realities, experiences and situations with which we must work in an inclusive, inclusive, equal and equitable manner. In this sense, working from the perspective of genderin schools make sus question the different stereotypes of cultural roles with which we have grown and we have been educated, as well as it opens the possibility of developing new or improving the existing contents of socialization and relation ship between people in favor ofequality. Therefore, our research revolves around the analysis of the gender perspective reflected in the educational projects of four public schools, from a comparative perspective, in order to identify which model of attention to the gender perspective is present in its regulations educational, through a documentary analysis of content as a qualitative research methodology. In short, this study allows us to approach certain realities to improve the quality of teaching practice, move towards the goals of an intercultural education with a gender perspective and transform the role of the school in our plural society.

Palabras clave: perspectiva de género, educación, normativa escolar, igualdad.

Keywords: gender perspective, education, school regulations, equality.

\section{LA PERSPECTIVA DE GÉNERO EN INSTITUCIONES EDUCATIVAS}

Desde hace décadas se reflexiona que las escuelas, además de ser un espacio privilegiado para transformar cuestiones sobre desigualdades de género, normalmente en la actualidad podemos decir que se perpetúa manteniendo el binomio del discurso sexual y de la complementariedad de los géneros. Esto se cristaliza en el diseño curricular, en el contenido de los libros de textos, en las actitudes del profesorado, en las elecciones de itinerarios, en la feminización de la gestión escolar frente a la masculinización de los cargos directivos, etc. (Rodríguez Martínez, 2011 en Calvo y Picazo,2016). 
En el proceso de desigualdad de género, debemos tener presente que la identidad femenina y/o masculina es el resultado de una construcción biológica, psicológica y social inmersa en una estructura sociocultural que, a la vez, fluye e influye en la educación. Por este motivo, según Tamayo Garza (2016) este proceso de construcción se considera dinámico.

De acuerdo a esta misma autoría, las representaciones sociales manifestadas a través de valores,normas, tradiciones, costumbres... son intrínsecas y pasan a convertirse en significancia simbólica aferrada a la subjetividad del contexto histórico en función a experiencias y circunstancias vitales.Sin embargo, la identidad de género está en un proceso de resignificación de valores y normas, apoyándose en el aprender a reflexionar y aprender a ser de acuerdo al contexto sociocultural en donde a las mujeres siempre han estado subordinadas por el hombre (Tamayo Garza, 2016).

En este proceso de construcción de la identidad, juega un papel muy importante la educación, a través de la socialización como elemento clave para destruir desigualdades. Pues, los procesos de aprendizaje permiten que podamos asumir valores y normas implícitas en nuestro grupo de referencia y adaptarnos a su forma de vida. Asimismo, es en el aprendizaje donde debe reinar una educación crítica capaz de actuar sobre la posición de poder del hombre hacia la mujer que sigue suscitando discriminaciones en diversas facetas de la vida, así como, incluso, llegando a episodios de violencia (Lorente, 2007 en Moreno Sánchez y Márquez Vázquez, 2016).

En el proceso de aprendizaje de los/as menores, según García Perales (2012), se empieza a reconocer a sí mismos/as y se empieza a diferenciar qué acciones son las adecuadas según el género, considerándose por adecuado las disposiciones que reproducen el comportamiento ya sea masculino y/o femenino en una determinada cultura en la que se desarrollan. Es en lo cultural donde se dificulta la percepción de las actitudes sexistas y, por ello, se transmiten inconscientemente. En este sentido, las valoraciones ante el género deben ser reflexionadas, analizadas y evaluadas en la práctica educativa para mejorar la calidad de la enseñanza y tomar decisiones con el fin de avanzar en prácticas socioeducativas y culturales más igualitarias.

Por su parte, Tamayo Garza (2016) expone que la educación, también como elemento socializador, no está al margen de la cultura y sus procesos, sobre todo, cuando es parte de la transformación social en pro de una mayor igualdad y justicia. En la misma línea, las instituciones educativas son transmisoras de normas y valores, de ideología y, por tanto, de las representaciones sociales respecto al género. Los/as profesionales de la educación, de acuerdo a esta autoría, transmiten de forma inconsciente su pensamiento ideológico y, con él, contribuyen a la construcción del género en el ámbito educativo.

[...] La práctica pedagógica cumple con la función social determinada por las condiciones políticas, económicas, sociales y culturales. La escuela, al transferir casi intactos los valores de la familia, reproduce el sexismo de la misma manera que lo hace el medio social: al transferir el orden social en los valores y las normas, se transfiere de manera no visible, las diferencias de género dejando a las mujeres en los lugares menos favorecidos para su desarrollo y se promueve la discriminación (aunque no únicamente de género (Tamayo Garza, 2016, 741).

\section{MÉTODO Y METODOLOGÍA DE INVESTIGACIÓN}

El objetivo es analizar qué se entiende por perspectiva de género en los proyectos educativos de cuatro centros escolares públicos desde una mirada comparativa. Concretamente, exponemos los resultados obtenidos sobre qué modelo de atención a la perspectiva de género está presente en las normativas educativas.

Investigación enmarca en las Ciencias Sociales. Su método es el estudio de caso múltiple, ya que indagamos, de forma sistemática, acerca del conocimiento y comprensión de fenómenos socioculturales y educativos en dos centros escolares de infantil y primaria de la provincia de Sevilla (España), en paralelo con dos centros escolares de inicial y primaria de la provincia de Salta (Argentina). En este sentido, la mirada comparativa, nos ha posibilitado un elemento contextual para enriquecer el análisis de los datos hacia una valoración más completa. Recordamos que la finalidad de este estudio de caso múltiple es conocer las particularidades propias de cada caso escolar para alcanzar una 
mayor comprensión de las categorías a analizar, y por ello, la representatividad no está presente en esta investigación. Acerca de las decisiones muestrales, las escuelas han sido seleccionadas según los criterios de la investigadora, de forma deliberada y no aleatoria, ni probabilística. Los criterios comparativos están basados en la posibilidad de acceder a centros educativos públicos de infantil y primaria, con alumnado mixto y presencia de alumnado de origen extranjero, originario y/o de minorías étnicas, ubicados en zonas urbanas de las provincias objeto de estudio y con antecedentes sobre prácticas relacionadas con la perspectiva de género.

Con respecto al diseño de la investigación, este estudio de caso múltiple se ubica en un diseño exploratorio, descriptivo, explicativo-interpretativo y evaluativo.

Desde una metodología cualitativa, la técnica aplicada ha sido el análisis documental institucional de los proyectos educativos cuyo descriptor es Perspectiva de Género (CA.PG). Para ello se ha utilizado una ficha de contenido como instrumento de recogida de datos. El análisis de datos se realizó de forma no frecuencial y a través del discurso sobre la categoría de estudio. Además, en general, se ha utilizado como principal herramienta de análisis el sistema categorial, que junto con la codificación de la información nos ha posibilitado la organización, descripción e interpretación de todos los datos recopilados sin la necesidad de apoyarnos en programas informáticos cualitativos.

\section{DISCUSIÓN}

Reflejamos la comparación de la información obtenida a partir de los indicadores comparativos enfunciónalacategoríaPerspectivadeGénero(CA.PG), utilizados para realizar la comparación de los proyectos educativos de los centros escolares por contexto de estudio. Estos son:

- Consideración sobre la perspectiva de género.

- Acciones descritas en el proyecto educativo relacionadas con la perspectiva de género. En comparación con el resto de los centros escolares, la Escuela Indalecio Gómez (Ca1) es en el único centro escolar donde no se especifica la terminología perspectiva de género en su proyecto educativo, pero se relaciona las cuestiones de género con la educación sexual'. En este sentido, solo se menciona la diferencia de sexos en el apartado sobre la Educación Sexual Integral y en el uso de un lenguaje no sexista, en ocasiones, en el discurso de la normativa escolar.

Por otra parte, tanto en la Escuela Roberto Romero (Ca2) de Salta como en los centros escolares de Sevilla, sí se recoge en su normativa escolar la perspectiva de género.

En la Escuela Roberto Romero (Ca2) aparece la perspectiva de género en el contenido curricular de formación ética y ciudadana, cuya competencia a adquirir por el alumnado es el reconocimiento de los prejuicios relacionados con el rol de ambos géneros y con situaciones relacionadas con la igualdad. También, podemos ver la relación de la perspectiva de género en la visión social de esta escuela en la que se expone una formación integral y permanente de la mujer y el hombre en sus diversas dimensionas, guiados por valores de justicia, igualdad, libertad y solidaridad.

En la misma línea están las escuelas sevillanas. En primer lugar, en el CEIP San José Obrero (Ca3) sí aparece la perspectiva de género en el proyecto educativo como línea de actuación pedagógica a partir de metodologías con carácter activo, motivador y participativo, partiendo de los intereses del alumnado, favoreciendo el trabajo entre iguales y el uso de la perspectiva de género en la vida cotidiana y en el entorno inmediato. También se considera, en este centro escolar, la perspectiva de género desde la transversalidad, sobre todo, en las áreas de educación en valores desde la superación de las desigualdades por razón de género y en el contenido curricular desde la integración de la igualdad de género: detección de situaciones discriminatorias, prejuicios culturales, estereotipos de género, participación equitativa, reflexión del significado y consecuencia de la violencia contra las mujeres, medidas preventivas...

\footnotetext{
${ }^{1}$ Se identifica la perspectiva de género como eje principal en el marco del Proyecto de Educación Sexual Integral (ESI), como acción específica por normativa nacional argentina.
} 
Por su parte, en el CEIP Andalucía (Ca4), también se recoge la perspectiva de género en su proyecto educativo haciendo referencia al respeto de las diferencias y a la diversidad de identidades no sujetas a roles de género tradicionales. El género es tratado en este centro escolar desde la lucha contra situaciones de discriminación y desigualad social, desde la igualdad de trato y oportunidades entre hombres y mujeres a nivel académico y profesional evitando los estereotipos y prejuicios sexistas y desde la eliminación de conductas violentas por patrones culturales de género.

Con respeto a las acciones relacionadas con la perspectiva de género que se llevan a cabo en loscentrosescolaresresaltamosqueenlasescuelassalteñasserelacionaconlasaccionesque se implementan desde el proyecto educativo de Educación Sexual Integral (ESI), valorando positivamente la diversidad de cada persona. La ESI como línea de intervención socioeducativa para profundizar en políticas socioeducativas y en políticas de cuidado de la infancia y la adolescencia.Derechoaconvivireninstitucioneseducativasenlasqueserespetenporiguallos derechos entre hombres y mujeres, sin estereotipos de género que cimente desigualdad. Dos ejes temáticos: reconocimiento de la perspectiva de género y respeto a la diversidad. Además, concretamente, en la Escuela Roberto Romero (Ca2) se trabaja la perspectiva de género en las actividades curriculares.

En comparación con el contexto sevillano, los centros escolares trabajan la perspectiva de género en acciones tanto internas como externas de la institución, según la norm ativa escolar. En el CEIP San José Obrero (Ca3), se recoge la perspectiva de género en el Plan de convivencia de acuerdo a al protocolo de actuación y medidas para la detección y atención de casos de violencia de género en el ámbito educativo. En este sentido, la violencia de género se entiende como manifestación de la discriminación, la situación de desigualdad y las relaciones de poder de los hombres hacia las mujeres que se ejerce sobre estas por el hecho de serlo. Por otra parte, también se recoge en el Plan de Igualdad como instrumento para diseñar acciones que potencien la igualdad de género. En el marco de este plan de igualdad, la Comisión de coeducación es la encargada de la visibilización de un lenguaje no sexista, realización de un diagnóstico de coeducación, nivel de participación, conflictividad... También, concretamente, en el Plan de Acción Tutorial se trabaja el desarrollo personal y social a través de roles, estereotipos, valores, identidad, expectativas, emociones, relaciones interpersonales, no violencia, educación afectivo-sexual, aceptación de críticas y elogios, toma de decisiones, etc. Por último, se destaca actividades relacionadas con efemérides y actividades puntuales como charlas, exposiciones, murallas y talleres.

En el CEIP Andalucía (Ca4) la perspectiva de género también se recoge en acciones internas y externas al centro escolar, como son el Plan de Convivencia a través de la comisión de convivencia que es la encargada de realizar un análisis del clima de convivencia del centro escolar, teniendo en cuenta las diferencias propias de la sociabilización de género. Y el Equipo de Coeducación, cuya función principal es la sensibilización de la igualdad entre hombres y mujeres en toda la comunidad educativa. Como actividades externas donde participa toda la comunidad educativa, destacamos actividades relacionadas con efemérides, proyectos socioeducativos en los que se colabora con asociaciones que trabajan en materia de género y acciones puntuales como charlas, exposiciones, murales, talleres...

\subsection{Modelo de atención a la perspectiva de género}

En la Escuela Indalecio Gómez (Ca1) de Salta dándole respuesta a la categoría de estudio, de qué manera se atiende y se gestiona la perspectiva de género, se puede concluir que aunque no se considera trabajar la perspectiva de género de forma práctica, se mantiene un discurso teórico con enfoque de igualdad entre hombres y mujeres, al menos recogido así en la normativa escolar por la Ley de Educación Sexual Integral (ESI). En este sentido, concluimos que la perspectiva de género se considera desde un modelo educativo de igualdad y coeducación más bien desde la teoría.

La Escuela Roberto Romero (Ca2) de Salta a pesar de no trabajar en profundidad la perspectiva de género se promueve un enfoque de igualdad entre hombres y mujeres. En este sentido, concluimos que la perspectiva de género se considera desde un modelo educativo de igualdad y coeducación, también más cercano a la teoría que a la práctica.

En el CEIP San José Obrero (Ca3) de Sevilla se gestiona la perspectiva de género con enfoque de igualdad para la superación de las desigualdades por razón de género, tanto en la teoría como en la práctica, desde la concienciación ante situaciones discriminatorias y prejuicios de género, desde la reflexión de las consecuencias sobre la violencia de género, desde el fomento de la 
igualdad, corresponsabilidad, autonomía, solidaridad, participación social... alejados de modelos de masculinidad tradicionales.

Por último, en el CEIP Andalucía (Ca4) de Sevilla se atiende a la perspectiva de género con enfoque de igualdad, respetando las diferencias y la diversidad de identidades no sujetas a roles de género tradicionales, superando así las desigualdades de forma teórica y práctica a través de la implicación docente, la evaluación de actividades, el conocimiento y análisis de situaciones discriminatorias...

Tabla 1: Comparativa de la Perspectiva de Género según los proyectos educativos de los centros escolares por contexto de estudio.

\begin{tabular}{|c|c|c|c|c|}
\hline \multirow{2}{*}{ CA.PG } & \multicolumn{4}{|c|}{ PROYECTOS EDUCATIVOS } \\
\hline & \multicolumn{2}{|c|}{ ESCUELAS DE SALTA (Argentina) } & \multicolumn{2}{|c|}{ ESCUELAS DE SEVILLA (España) } \\
\hline $\begin{array}{l}\text { INDICADORES } \\
\text { COMPARATIVOS }\end{array}$ & $\begin{array}{c}\text { Caso 1- } \\
\text { Escuela Indalecio } \\
\text { Gómez }\end{array}$ & $\begin{array}{c}\text { Caso 2- } \\
\text { Escuela Roberto } \\
\text { Romero }\end{array}$ & $\begin{array}{l}\text { Caso 3- } \\
\text { CEIP San José } \\
\text { Obrero }\end{array}$ & $\begin{array}{l}\text { Caso 4- } \\
\text { Andalucía }\end{array}$ \\
\hline 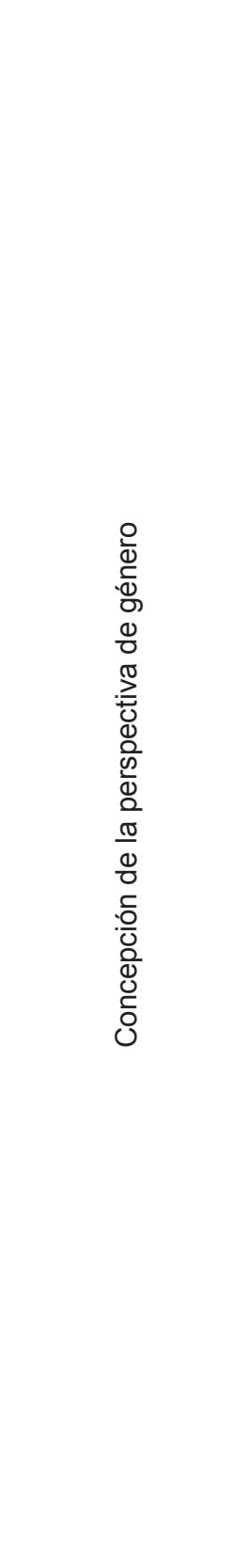 & $\begin{array}{c}\text { No se especifica nada } \\
\text { de la perspectiva de } \\
\text { género. } \\
\text { Se relaciona la } \\
\text { perspectiva de } \\
\text { género con la } \\
\text { educación sexual. } \\
\text { Solo se menciona la } \\
\text { diferencia de sexos en } \\
\text { el apartado sobre la } \\
\text { Educación Sexual } \\
\text { Integral (ESI) y en el } \\
\text { uso de lenguaje no } \\
\text { sexista, en ocasiones. }\end{array}$ & $\begin{array}{c}\text { Sí se recoge en el } \\
\text { contenido curricular de } \\
\text { formación ética y } \\
\text { ciudadana: reconocer } \\
\text { los prejuicios } \\
\text { relacionados conel rol } \\
\text { de ambos género y } \\
\text { situaciones } \\
\text { relacionadas con la } \\
\text { igualdad. } \\
\text { Visión social de la } \\
\text { escuela: formación } \\
\text { integral y permanente } \\
\text { de la mujer y el } \\
\text { hombre en sus } \\
\text { diversas dimensiones, } \\
\text { guiados por valores de } \\
\text { justicia social, } \\
\text { igualdad, libertad, } \\
\text { solidaridad. }\end{array}$ & $\begin{array}{l}\text { Sí se recoge en el } \\
\text { PEC como línea } \\
\text { general de actuación } \\
\text { pedagógica a partir } \\
\text { de metodologías con } \\
\text { carácter activo, } \\
\text { motivador y participa- } \\
\text { tivo, que parta de los } \\
\text { intereses del } \\
\text { alumnado, favorez- } \\
\text { ca el trabajo entre } \\
\text { iguales y el uso de la } \\
\text { perspectiva de género } \\
\text { en la vida cotidiana y } \\
\text { en el entorno } \\
\text { inmediato. } \\
\text { Tratamiento } \\
\text { transversal en las } \\
\text { áreas de educación } \\
\text { en valores desde la } \\
\text { superación de las } \\
\text { desigualdades por } \\
\text { razón de género. } \\
\text { Tratamiento transver- } \\
\text { sal en el contenido } \\
\text { curricular desde la } \\
\text { integración de la } \\
\text { igualdad de género: } \\
\text { detección de situacio- } \\
\text { nes discriminatorias, } \\
\text { prejuicios cultura- } \\
\text { les, estereotipos de } \\
\text { género, participación } \\
\text { equitativa, reflexión } \\
\text { del significado y } \\
\text { consecuencia de la } \\
\text { violencia contra las } \\
\text { mujeres, medidas } \\
\text { preventivas... }\end{array}$ & $\begin{array}{c}\text { Sí se recoge en el PEC } \\
\text { Respeto a las } \\
\text { diferencias y a la } \\
\text { diversidad de } \\
\text { identidades no sujetas a } \\
\text { roles de género } \\
\text { tradicionales. } \\
\text { El género es tratado } \\
\text { desde la lucha contra } \\
\text { situaciones de } \\
\text { discriminación y } \\
\text { desigualad social, desde } \\
\text { la igualdad de trato y } \\
\text { oportunidades entre } \\
\text { hombres y mujeres a } \\
\text { nivel académico y } \\
\text { profesional evitando los } \\
\text { estereotipos y prejuicios } \\
\text { sexistas y desde la } \\
\text { eliminación de conductas } \\
\text { violentas por patrones } \\
\text { culturales de género. }\end{array}$ \\
\hline
\end{tabular}




\begin{tabular}{|c|c|c|c|c|}
\hline \multirow{2}{*}{ CA.PG } & \multicolumn{4}{|c|}{ PROYECTOS EDUCATIVOS } \\
\hline & \multicolumn{2}{|c|}{ ESCUELAS DE SALTA (Argentina) } & \multicolumn{2}{|c|}{ ESCUELAS DE SEVILLA (España) } \\
\hline $\begin{array}{l}\text { Acciones relaciona- } \\
\text { das con la perspec- } \\
\text { tiva de género }\end{array}$ & $\begin{array}{l}\text { Proyecto de Educación } \\
\text { Sexual Integral (ESI). } \\
\text { Se valora positivamente } \\
\text { la diversidad de } \\
\text { cada persona. } \\
\text { La ESI como línea de } \\
\text { intervención socioedu- } \\
\text { cativa para profundizar } \\
\text { en políticas socioedu- } \\
\text { cativas y en políticas } \\
\text { de cuidado de la infan- } \\
\text { cia y la adolescencia. } \\
\text { Derecho a convivir en } \\
\text { instituciones educativas } \\
\text { en las que se respeten } \\
\text { por igual los derechos } \\
\text { entre hombres y mu- } \\
\text { jeres, sin estereotipos } \\
\text { de género que cimente } \\
\text { desigualdad. Dos ejes } \\
\text { temáticos: reconoci- } \\
\text { mientode la perspectiva } \\
\text { de género y respeto a la } \\
\text { diversidad }\end{array}$ & $\begin{array}{l}\text { Educación Sexual } \\
\text { (ESI) } \\
\text { Actividades } \\
\text { curriculares } \\
\text { Proyecto de }\end{array}$ & $\begin{array}{l}\text { Plan de convivencia: } \\
\text { protocolo de actuación y } \\
\text { medidas para la detec- } \\
\text { ción y atención decasos } \\
\text { de violencia de género } \\
\text { en el ámbito educativo. } \\
\text { Violencia de género } \\
\text { entendida como } \\
\text { manifestación de la } \\
\text { discriminación, la situa- } \\
\text { ción de desigualdad y } \\
\text { las relaciones de poder } \\
\text { de los hombres hacia las } \\
\text { mujeres que se ejerce } \\
\text { sobre estas por el hecho } \\
\text { de serlo. } \\
\text { Plan de lgualdad: ins- } \\
\text { trumento para diseñar } \\
\text { acciones que potencien } \\
\text { la igualdad de género. } \\
\text { Comisión de coeduca- } \\
\text { ción: visibilización de un } \\
\text { lenguaje no sexista, reali- } \\
\text { zación de un diagnóstico } \\
\text { de coeducación, nivel de } \\
\text { participación, } \\
\text { nos Actividades puntua- } \\
\text { les } \\
\text { conflictividad... } \\
\text { Plan de acción tutorial: } \\
\text { se trabaja el desarrollo } \\
\text { personal y social a través } \\
\text { de roles, estereotipos, } \\
\text { valores, identidad, expec- } \\
\text { tativas, emociones, rela- } \\
\text { ciones interpersonales, } \\
\text { no violencia, educación } \\
\text { afectivo-sexual, acepta- } \\
\text { ción de críticas y elogios, } \\
\text { tema de decisiones, etc. }\end{array}$ & $\begin{array}{l}\text { Plan de Convivencia: } \\
\text { la comisión de con- } \\
\text { vivencia es la encar- } \\
\text { gada de realizar un } \\
\text { análisis del clima de } \\
\text { convivencia del centro } \\
\text { escolar, teniendo en } \\
\text { cuenta las diferencias } \\
\text { propias de la } \\
\text { sociabilización de } \\
\text { género. Equipo de } \\
\text { coeducación: sensibili- } \\
\text { zación de la } \\
\text { igualdad entre hom- } \\
\text { bres y mujeres en } \\
\text { toda la comunidad } \\
\text { educativa Actividades } \\
\text { Efemérides Proyec- } \\
\text { tos socioeducativos } \\
\text { Actividades puntuales: } \\
\text { Charlas, exposiciones, } \\
\text { murales, talleres }\end{array}$ \\
\hline
\end{tabular}

Fuente: elaboración propia a partir de la información obtenida de los proyectos educativos de cada caso escolar.

\section{CONCLUSIONES}

Ante lo expuesto, consideramos pertinente que, en la actualidad, se trabaje en la atención a la diversidad de género desde una transformación social global, pues debemos conseguir convertir a los centros escolares como espacios en lucha contra las desigualdades vinculadas al género, pues consideramos que el cambio puede hacer a través de la educación en la que tienen acceso, por de- 
recho, todos/aslos/asmenores. En este cambio, es fundamental considerar todas las normativas con las que se cuenta a nivel internacional y nacional con el fin de educar para la igualdad entre hombres y mujeres (Calvo y Picazo, 2016).

En este sentido, consideramos importe nombrar al último Acuerdo de 16 de febrero de 2016, del Consejo de Gobierno de la Junta de Andalucía, por el que se aprueba el II Plan Estratégico de Igualdad de Género en Educación 2016 - 2021. Este plan constituido como instrumento de política educativa integral, se concibe como

[...] el marco de actuación y la herramienta para continuar impulsando la igualdad dentro del sistema educativo, tanto en aspectos estructurales y culturales de la Administración, como en los relacionados con la vida y las actuaciones de los centros docentes, contemplando, asi mismo, la diversidad de identidades de género, de orientaciones sexuales, de modelos de familia y de formas de convivencia, evitando cualquier tipo de discriminación por causa de las mismas. el diseño de las políticas a favor de las mujeres ha encontrado su desarrollo en los Planes de Igualdad que vienen a dar respuesta a la necesidad de conquistar derechos igualitarios para las mujeres, eliminando los obstáculos que se interponen a la igualdad real y al acceso al ámbito de lo público. El II Plan estratégico de lgualdad de Género en educación propone actuaciones en este mismo sentido a favor de los derechos de las mujeres y, además, incorpora actuaciones que incidan en la cultura real que sustenta la desigualdad, con actuaciones a favor de los hombres y de las mujeres en aquellas desigualdades específicas que produce la tradicional socialización diferenciada. De esta forma tanto las chicas como los chicos refuerzan los aspectos que, en cada caso, les son favorables y recuperan los que se les había prohibido, posibilitando su pleno desarrollo como personas (Acuerdo de 16 de febrero de 2016, del Consejo de Gobierno de la Junta de Andalucía, por el que se aprueba el II Plan Estratégico de Igualdad de Género en Educación 2016-2021, 14).

En la línea de la educación transformadora, como profesionales de la educación, debemos seguir cuestionando los estereotipos y prejuicios bajo los que nos educamos, denunciando la jerarquización de las disciplinas, reconociendo la estructura social en la que nos hemos educado y preguntándonos hacia dónde queremos llegar a través de la educación. En definitiva, debemos ser partícipes y generar participación, «donde el aprendizaje tenga que ver con una transformación interna de la persona» (Lobo y Fernández, 2016, 89).

A tenor de esto último, ¿qué se puede hacer desde las instituciones educativas? De acuerdo con Tamayo Garza $(2016,742)$, exponemos algunas de sus líneas aseguir.

- [...] - Poner atención en las formas del lenguaje hacia niños y niñas: frases como «una niña bonita no grita», «las niñas se portan mejor que los niños», "qué letra tan fea, bueno, eres niño», etc.

- Evitar condicionamientos de comportamiento por el género, por ejemplo: los niños pueden jugar con movimientos bruscos en el recreo; las niñas no juegan con los niños porque son débiles; los trabajos de exposición son tareas principalmente de las niñas porque saben decorar y hermosear el material; los niños compiten abiertamente en áreas como matemáticas y ciencias.

- Propiciar el pensamiento crítico, reflexivo y creativo en ambos géneros, propiciar el diálogo y el respeto. Buscar crecer junto a los otros y no contra los otros.

- Reconocer la sensibilidad y emotividad en ambos, así como la fuerza y capacidad de tomar decisiones en ambos.

Por su parte Calvo y Picazo $(2016,87)$, las líneas de avance sobre la perspectiva de género en el sistema educativo están vinculadas a temas concretos para la eliminación de las desigualdades vinculadas al género, entre los que destaca

- [...] - La elección de los estudios, con el fin de que hombres y mujeres se distribuyan equitativamente las diferentes actividades profesionales y se posicionen socialmente de manera más igualitaria.

- Las masculinidades, favoreciendo que los chicos asuman modelos más sanos, en los que no tengan que relacionarse mediante la violencia, en los que puedan expresar sus emociones, en los que puedan exceder la heterosexualidad, en los que no tengan que ocupar el papel de proveedores, etc. y así poder mantener también relaciones personales más igualitarias. 
- Las relaciones afectivos-sexuales, presentándoles desde la infancia modelos alternativos a los románticos (basados en la dependencia, en la complementariedad, en el sufrimiento...) y una perspectiva global de la sexualidad, no heterosexual ni coitocéntrica, que no limite ni perjudique, sino que contribuya al desarrollo personal.

En definitiva, para la atención a la diversidad de género, las escuelas públicas deben contar con Proyectos Educativos verdaderamente inclusivos, consensuados y asumidos por sus comunidades educativas, sensibilizadas y motivadas por la construcción de una sociedad más justa. Asimismo, se debe entender la diversidad de manera global, sin clasificar a las personas en dos grupos en función de su género y, por supuesto, superándose el binarismo sexual considerándose como «una muestra más de la riqueza humana» (Calvo y Picazo, 2016, 88).

\section{REFERENCIAS BIBLIOGRÁFICAS}

Acuerdo de 16 de febrero de 2016, del Consejo de Gobierno de la Junta de Andalucía, porel que se aprueba el II Plan Estratégico de Igualdad de Género en Educación 2016-2021. BOJA nº 41 (2016).

Calvo García, G.y Picazo Gutiérrez, M.(2016). La Diversidad de Género en la Escuela Pública y la exclusión que produce el binarismo. Revista Interuniversitaria de Formación del Profesorado, 30 (1),81-90.

García Perales, R. (2012). La educación desde la perspectiva de género. ENSAYOS, Revista de la Facultad de Educación de Albacete, 27, 1-18.

Lobo, C., y Fernández, S. (2016). Aplicación del Enfoque de Género en Trabajos de Investigación de alumnado universitario de Ciencia y Tecnología. IKASTORRATZA. e-Revista de Didáctica, 17, 89-110. Recuperado de http://www.ehu.es/ ikastorratza/17_alea/6.pdf.

Moreno Sánchez, E. y Márquez Vázquez, C. (2016). Determinantes implicados en la construcción de la violencia de género. El caso de las escuelas primarias en una provincia de España. Archivos Analíticos de Políticas Educativas, 24 (6). Recuperado de http://dx.doi.org/10.14507/epaa.v24.2132.

Tamayo Garza, J. (2016). Identidad de género de las maestras de primaria. Ponencia. Mujeres e investigación. Aportaciones interdisciplinares. VI Congreso Universitario Internacional Investigación y Género (p.731-744). Recuperado de https://idus.us.es/xmlui/handle/11441/51971. 